\title{
Die deutsche Bistumspresse von Analyse zu Analyse
}

\author{
von Michael Schmolke
}

Nicht nur Bücher, ganze Mediengattungen haben ihre Schicksale. Das gilt z.B. für die deutsche Bistumspresse. Kaum einer Gattung wurden so viele Untersuchungen und Analysen gewidmet wie ihr. Damit meine ich quantitative bzw. quantitativ-qualitative Untersuchungen mit mehrheitlich gemeinnütziger Zwecksetzung. Nun werden heute wohl alle Printmedien, die groß auf dem Markt sind - und die Bistumspresse war einmal groß auf dem Markt: 1963 war ihre kumulierte Auflage etwa zweimal so hoch wie die des "Stern" -, immer wieder analysiert, einerseits, um ihre Position im Markt möglichst genau zu erfassen, und andererseits, um sie dem Werbemarkt transparent, d.h. in Wirklichkeit: möglichst attraktiv zu machen.

Aber diese Untersuchungen dienen in der Regel nur dem einzelnen Verlagshaus, das sie in Auftrag gibt, und sie dienen dem Marketing auf verschiedenen Ebenen bis hin zu der Frage, wann ein Zeitschriftenobjekt eingestellt werden muß, ehe es Verluste einzufahren beginnt. (Ausschließlich aus diesem Grund werden z.B. aufwendig gestartete Neugründungen, in jüngerer Zeit etwa "Tango", wieder eingestellt: Aus genauer Marktbeobachtung weiß der Verlag, daß sein Objekt die Planungs- und Start-Investitionen nicht schnell genug wieder einspielen wird.)

Die hier gemeinten Untersuchungen der Bistumspresse sind jedoch nicht betriebswirtschaftlich- bzw. marketing-orientierte (die es auch gegeben hat), sondern insofern "gemeinnützige", als nicht nur die ganze - wenn auch nicht immer vollzählige - Gruppe der Verlage/ Blätter daran interessiert war, sondern die hinter den Titeln stehenden Diözesen und damit eigentlich "die Kirche", insofern sie in diesen Wochenblättern ein Instrument der Pastoral sah und sieht.

Denn die Bistumsblätter, auch Kirchenzeitungen, Kircherıblätter, Diözesanblätter genannt, sind nicht Wochenzeitungen wie viele andere neben ihnen, sondern zwar nicht offizielle, aber doch offiziöse oder mindestens halb-offiziöse Organe, deren Herausgeber de jure oder de facto in der Regel der Ortsbischof ist. Sie müssen nicht im Eigentum der Diözese stehen, sind ihr aber doch in sehr enger Bindung zugeordnet, was übrigens mit ihrer Entstehung unter den Konditionen der

Prof. Dr. Michael Schmolke ist Ordinarius für Kommunikationswissenschaften an der Universität Salzburg und Mitherausgeber dieser Zeitschrift. 
nationalsozialistischen Presselenkung zu tun hat. ${ }^{1}$ Sie sind außerdem Monopolblätter, d.h. ihr Verbreitungsgebiet ist ihre Diözese, und schon in der Nachbardiözese bekommt man sie nur auf besonderen Wunsch. Dieser Status hat - neben anderen Konditionen - die für den redaktionellen wie für den verlegerischen Bereich fatale Vorstellung entstehen lassen: Wenn uns in unserem monopolähnlichen Verbreitungsgebiet alle katholischen Haushalte abonnieren - und selbst wenn es nur 20 oder $10 \%$ tun, dann sind wir gesichert für alle Ewigkeit oder jedenfalls so lange, wie es katholische Haushalte gibt.

So stand dann auch hinter allem Untersuchungsaufwand, den man dieser Pressegattung als Gattung zugewandt hat, nicht die Frage nach betriebswirtschaftlicher Sinnhaftigkeit einzelner Verlagsobjekte, sondern die Frage nach existenzieller Absicherung unter schlechter werdenden Rahmenbedingungen. Von jeder neuen Studie wurde "irgendwie" das.Schlüsselwort für das Ende der Talfahrt ${ }^{2}$ erwartet. Dabei kamen seit den siebziger Jahren durchaus auch MarketingÜberlegungen zum Zuge, die jedoch nie so recht professionell ausformuliert und schon gar nicht zuende gedacht wurden, weil das Ende als solches nicht vorstellbar ist oder doch niemals offen als eine Vorstellbarkeit angesprochen wird.

Auch die jüngste (in meinem Sinne "gemeinnützige") Untersuchung "Chancen für die Bistumszeitungen 1994", die in Heft 2/1996 von "Communicatio Socialis" unter verschiedenen Aspekten von Rüdiger Schulz, Renate Köcher, Hans Mathias Kepplinger/Simone Christine Ehmig und Heiko Klinge dargestellt, interpretiert und mit kritischen Anmerkungen, aber auch Vorschlägen ausgestattet wurde, ist keineswegs frei von spero contra spem-Elementen. Das liegt dieses Mal gewiß nicht an den Machern der Untersuchung. Den sozialempirischen Teil hat das Institut für Demoskopie Allensbach durchgeführt und die vertiefende Inhaltsanalyse (Form und Inhalt der Bistumspresse) mit H.M. Kepplinger einer der auf diesem Gebiet erfahrensten Kommunikationswissenschaftler des deutschen Sprachraums (Institut für Publizistik der Universität Mainz). Dennoch überträgt sich sowohl aus Formulierungen der Allensbach-Studie als auch aus den Interpretationen in CS 2/1996 immer wieder die Anmutung: Man wird diese Zeitungen schon nicht untergehen lassen. Daß Heiko Klinge sein Aktionsprogramm ${ }^{3}$, das im übrigen längst angelaufen ist, so entschieden formuliert und auf der Basis der Medien-Dienstleistung Gesellschaft auch entsprechend fun-

1 Vgl. M. Hüsgen, Die Bistumsblätter in Niedersachsen während der nationalsozialistischen Zeit, Hildesheim 1975, 121-123.

2 Die Auflage der deutschen Bistumspresse hat sich seit ihrem Gipfelpunkt im Jahre 1963 (2,456 Mio. [tatsächlich verbreitete]) fast halbiert: 1,291 Mio.(verkaufte; 1995).

3 Hilfen zur Umsetzung der Erkenntnisse aus der Kommunikatoren- und Rezipientenbefragung und der Inhaltsanalyse, in: CS 29 (1996) 234-237. 
diert und ausgestattet hat, spricht Bände. Wenn er "hohe Anstrengungen von allen Beteiligten" einfordert, weiß er, wovon er spricht, denn auch nach der sog. "Feldbefragung" von 1975 wurden eine Reihe von Umsetzungsveranstaltungen ${ }^{4}$ durchgeführt, die nicht schlecht besucht waren und auf denen viel diskutiert wurde. Aber die Grundstimmung damals läßt sich nach meiner Erinnerung etwa so zusammenfassen: Es ist schon richtig, daß mit unseren Zeitungen nicht alles zum besten steht, aber uns [damit war, etwa aus der Sicht eines Chefredakteurs, das jeweils eigene Blatt gemeint] geht es ja eigentlich noch ganz gut, - nur keine Panik! Die Verleger hielten sich im allgemeinen bedeckt, und die Bischöfe als Herausgeber waren 1975 - im Unterschied zur AllensbachStudie - gar nicht ernsthaft befragt worden. Einiges von der 1975erHaltung scheint überlebt zu haben

- sowohl im Sinne der Bewältigung kognitiver Dissonanzen: „Die Chefredakteure sind stärker als Bischöfe und Verleger von der redaktionellen Leistung der Bistumspresse, das heißt ihrer eigenen Leistung, überzeugt ... So erwarten $47 \%$ der Chefredakteure, aber nur $23 \%$ der Verleger und $18 \%$ der Bischöfe, daß die Bistumszeitung dem überwiegenden Teil der Leser, großen persönlichen Nutzen bietet'“" Und: „Verleger plädieren deshalb entschiedener als Bischöfe und Chefredakteure für viele grundlegende Veränderungen, während sich viele Bischöfe und Chefredakteure mit ,Anpassungsmaßnahmen' begnügen wollen. “5

- als auch beim freundlichen Zuspruchstil der Analysatoren: „All dies sind wichtige Hinweise, daß die Säkularisierungstendenzen keineswegs alleiniger Grund für die Abwendung sind, daß die Kirchenzeitungen durch gemeinsame Anstrengungen von Redaktion und Verlag optimiert werden können und sich der Verfall der Bistumspresse nicht zwangsläufig fortsetzen muß."6

$\mathrm{Da}$ ich mich selber im Verdacht habe, dieser Hoffnung ebenfalls anzuhängen, werde ich im folgenden versuchen, einige Argumente zusammenzutragen, die die Gesamtentwicklung auf der Grundlage früherer Untersuchungen als einen durchgehenden Trend erkennbar machen, der seit mehr als einem Vierteljahrhundert vorhersehbar war und tatsächlich auch vorhergesehen wurde.

4 Vgl. M. Schmolke, Kommentar zu wichtigen Ergebnissen der Feldbefragung, in: Feldbefragung. Kommunikations- und Informationserwartungen der katholischen Bevölkerung gegenüber kirchlichen Massenmedien, insbesondere der Kirchenpresse, Teil D, Wuppertal 1975, 1-46, 1.

5 Chancen für die Bistumszeitungen. Bd. I: Situationsanalyse, Empfehlungen, 76.

6 Ebd., 38. 


\section{Seit 30 Jahren Niedergang ohne Untergang}

Rüdiger Schulz hat zur Vorbereitung und Interpretation die wichtigsten Vorgänger-Studien herangezogen und dokumentiert.? Sein Interesse war auf die Gewinnung vergleichbarer Daten gerichtet, und dafür standen Resultate aus 20 Jahren zur Verfügung. Aber schon deutlich vor der "Feldbefragung" von 1975 gab es zwei Studien, in denen die Eckwerte der drohenden Talfahrt erforscht, belegt und in gebührender Deutlichkeit einerseits der interessierten Öffentlichkeit (1970/71) und andererseits in vertraulicher und deshalb dringlicherer Form dem Auftraggeber Deutsche Bischofskonferenz (1973) mitgeteilt wurden.

Es handelt sich dabei im ersten Falle um Manfred P. Beckers fünfteilige Aufsatzfolge „Die Bistumspresse in der Bundesrepublik Deutschland“, die 1970 und 1971 in dieser Zeitschrift publiziert wurde ${ }^{8}$, im zweiten Falle um die Stellungnahmen Nr. 7, Nr. 11 sowie resümierend um die Kapitel 3.2 und 3.3 der "Zusammenfassung " des von der Deutschen Bischofskonferenz in Auftrag gegebenen "Gutachtens Katholische Publizistik 72/73“.9

Schon aus Beckers Studie, die übrigens einer Anregung des als besonders konservativ geltenden Franz Kroos (Chefredakteur von "Kirche und Leben", Münster/W.) zu verdanken ist, gehen wesentliche Indikatoren hervor, die in der folgenden Bistumsblatt-Forschung immer wieder aufgegriffen wurden:

1. Die, Talfahrt'

Während die Bistumsblatt-Gesamtauflage 1950 bis 1963 um jährlich $1,4 \%$ gestiegen war, sank sie seither (gemeint ist hier: bis 1969) um jährlich $1,5 \%$.

2. Die Bezieherdichte

Die Bezieherdichte ist ,nur' ein Meßwert; sie setzt rein rechnerisch die jährliche Gesamtauflage ins Verhältnis zur Zahl der Katholiken in der Bundesrepublik bzw. in der jeweiligen Diözese. 1963 waren 9,2 $\%$ der katholischen Bundesbürger Bezieher eines Bistumspresseprodukts, 1969 nur noch 7,8 \% (1990: 5,3\%10).

7 R. Schulz, Chancen für die Bistumszeitungen, in: CS 29 (1996) 153-205, 154, Anm. 4-6.

8 Vgl. CS 3 (1970) 299-310, 391-396; 4 (1971) 86-92, 182-195 und 281-291.

9 Auftragnehmer war der Autor dieses Aufsatzes, der damals einer der drei Direktoren des Deutschen Instituts für wissenschaftliche Pädagogik (Münster/W.) war und mit den Ressourcen dieses Instituts arbeiten konnte. Die aus dem Forschungsauftrag hervorgegangenen Texte sind bislang unveröffentlicht.

10 Vgl. M. Schmolke, Anfang der Talsohle, in: CS 26 (1993) 389-393. 
3. Die Nicht-Übereinstimmung bestimmter Merkmale der BistumsblattLeserschaft mit den Durchschnittswerten der westdeutschen Bevölkerung.

Die von Becker beschriebenen abweichenden Merkmale der Bistumsblatt-Leser/-Bezieher waren Wohnort, Bildung und Alter; dabei stützte er sich übrigens auf eine von der Arbeitsgemeinschaft Katholische Presse in Auftrag gegebene Leseranalyse11, die 1969/70 von Marplan durchgeführt wurde: $60 \%$ der Leser wohnten in Orten bis zu 20.000 Einwohnern (Bundesbürgerdurchschnitt damals: $29 \%$ ); Volksschule ohne Lehrabschluß (also den untersten Formalbildungsgrad) finden wir bei $39 \%$ der Leser, aber nur bei $30 \%$ im Bundesdurchschnitt. Und schließlich das Alter: die Gruppe der 20- bis 29jährigen sei deutlich unter- $(9,3 \mathrm{zu} 16,5 \%)$, die der über 60 jährigen deutlich überrepräsentiert $(32,4 \mathrm{zu} 22,5 \%)$.

Mit diesen drei Informationskomplexen, die fortan alle wesentlichen Untersuchungen durchziehen, waren Signale gesetzt, die schon bei einmaliger Bestärkung als Warnzeichen hätten verstanden werden können. Die Bestärkung folgte 1972/73 in dem oben erwähnten Gutachten für die Deutsche Bischofskonferenz. In dessen allgemeiner Untersuchung „Die Entwicklung der Bistumspresse in den Diözesen der Bundesrepublik Deutschland 1963-1972" (= Stellungnahme Nr. 7, April 1973) wurde die Becker-Studie mit dessen Hilfe fortgeschrieben und verfeinert. Sie mündete u.a. in folgenden Thesen:

"(1) Die Bistumspresse steckt zur Zeit [1973] in einer essentiellen Krise, die ihren Ursprung unter anderem im Rückgang der allgemeinen Kirchlichkeit hat. Ihr fehlen jüngere Leserkreise, ihre Verbreitung in den Gemeinden über 20.000 Einwohnern ist unterdurchschnittlich. Diese Entwicklung ist zwar auch bei den vergleichbaren evangelischen Kirchengebietsblättern feststellbar, doch bei der Bistumspresse in einem verstärkten Umfang bemerkbar.

(2) Die Bistumspresse hat seit 1950 noch nie derartig geringe relative Verbreitung gefunden wie in der Gegenwart. Sie erreicht zur Zeit anteilig weniger Leser als 1950.

(3) Anhand des Untersuchungsmaterials konnte in keinem Falle ein Anzeichen für eine positive Entwicklung in naher oder ferner Zukunft festgestellt werden.

(4) Die Nicht-Übereinstimmungen mit dem Bevölkerungsdurchschnitt bei den Merkmalen ,Alter' und 'Wohnortgröße' der Leser und der Kirchgänger verweisen - weil sie eben nicht nur typisch sind für die

11 AKP (Hg.), Reichweite und Leserschaftsstrukturen der katholischen Bistumspresse. 
Bistumspresse-Leser, sondern die Katholiken insgesamt - auf eine mindestens nach Alter und Wohnortgröße ,gestaffelte Kirchlichkeit* der Katholiken insgesamt, die sich bei den Lesern der Bistumspresse widerspiegelt. Die Faktoren, die etwa zu einer überdurchschnittlichen Kirchlichkeit in den kleineren Orten führen (verstärkte soziale Kontrollmöglichkeit), sind nicht zukunftsweisend (Stichwort: ,katholisches Milieu').“

\section{"Altersspagat" und andere Probleme}

Der wiederholte und bestärkte Überalterungsbefund veranlaßte die Auftraggeber, eine Präzisierung anzuregen, die schon im September 1973 als Stellungnahme Nr. 11 (Zur Altersstruktur der Leserschaft konfessioneller Presse) vorgelegt werden konnte. Sie stützte sich auf sieben zuvor entstandene Altersstrukturuntersuchungen, wovon sich zwei auf die überkonfessionelle Fachgruppe "Konfessionelle Presse“ bezogen (1961/62; 1971), eine auf die Bistumspresse (1969/70), eine auf die evangelischen Kirchengebietsblätter (1970), je eine auf ein evangelisches Kirchengebietsblatt (1967), ein Bistumsblatt (1972) und ein Familienblatt (1968).

Es wurde nach der 40-Jahres- und der 50-Jahres-Grenze gefragt. Die beiden folgenden Grafiken demonstrieren den Altersüberhang, wie er sich (A) für die Kollektive und (B) für die drei Einzeltitel schon

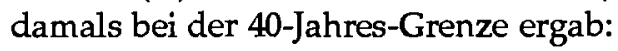




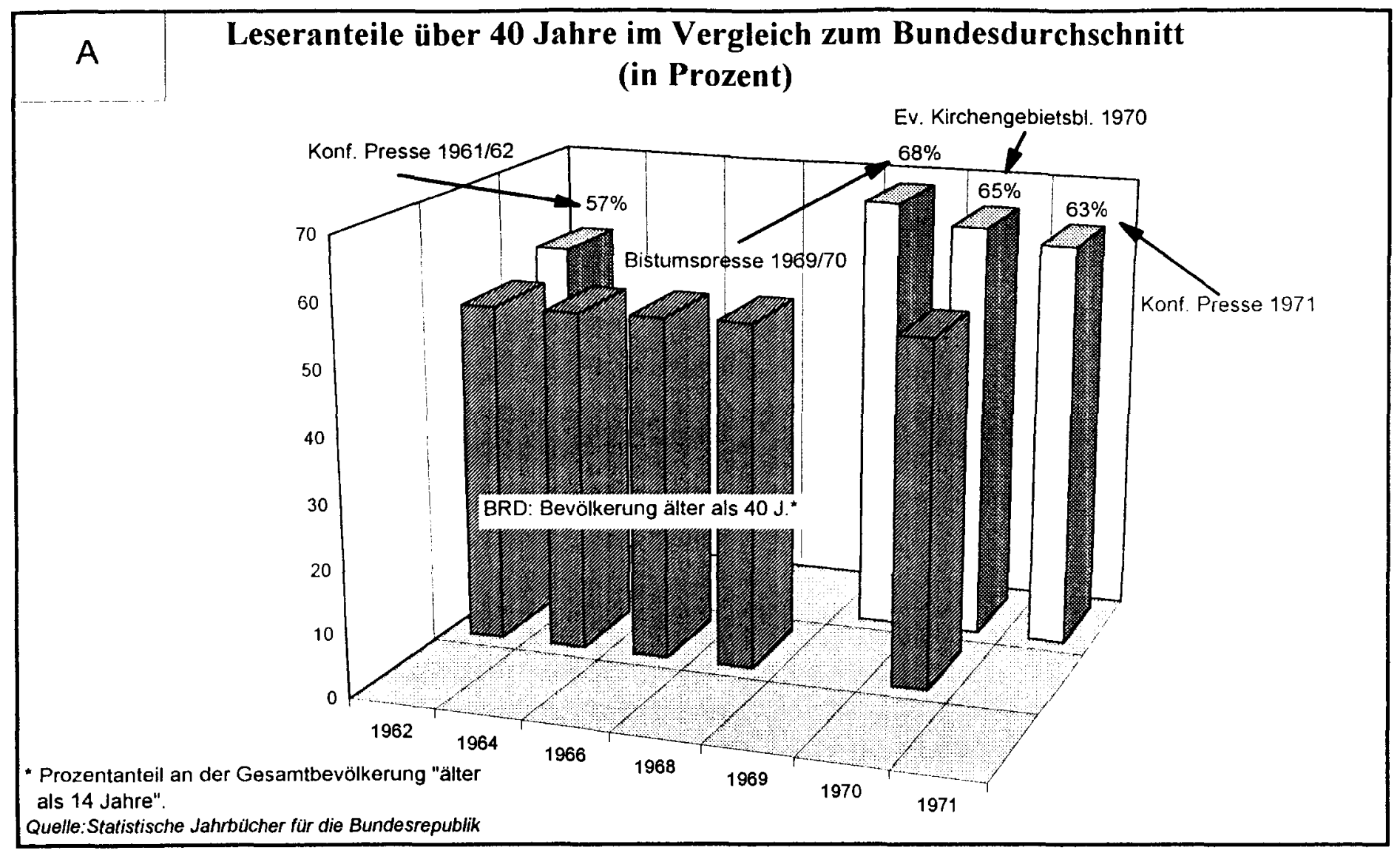




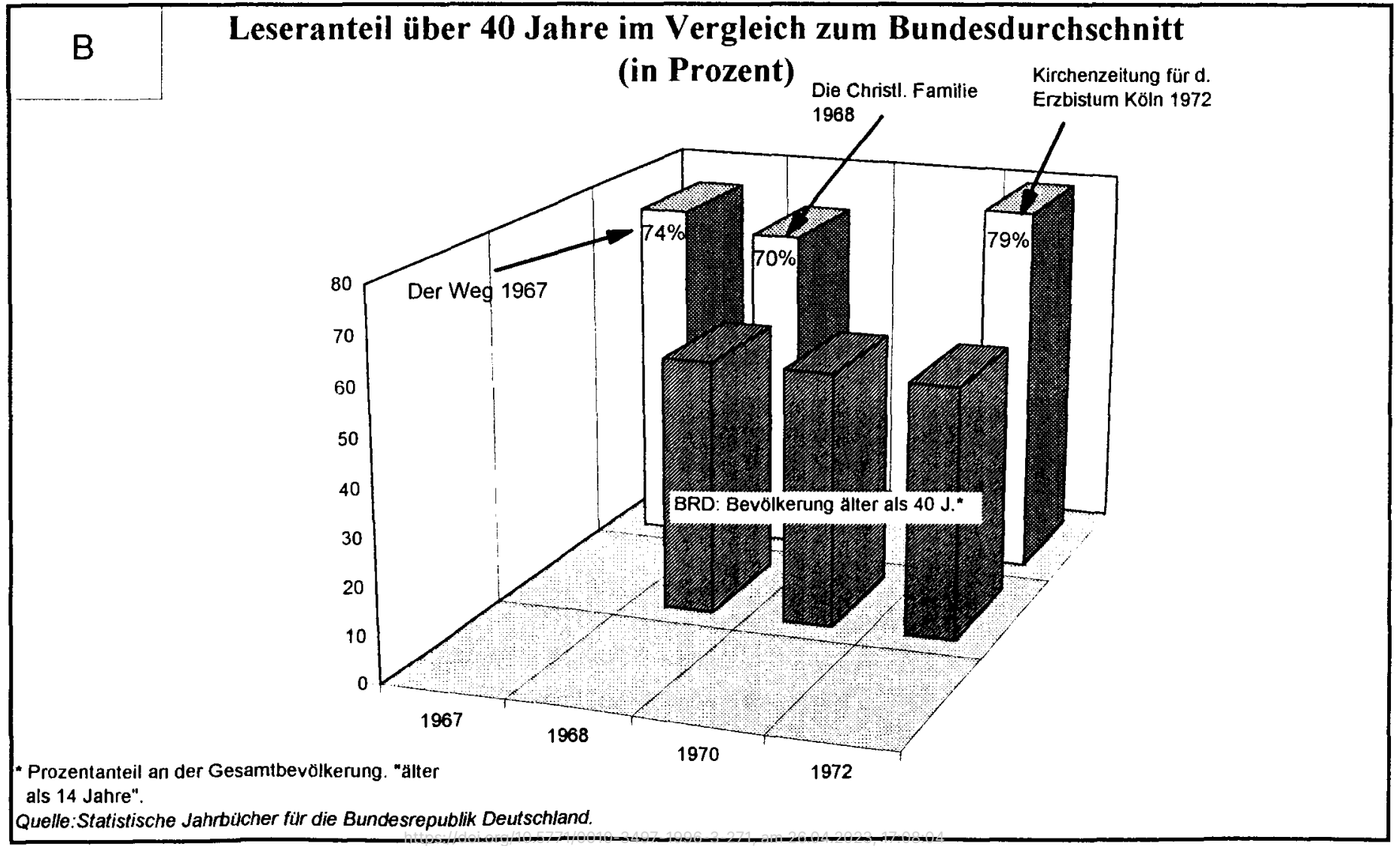


Angesichts dieser Vergleiche erlaubte ich mir die folgende Zusammenfassung: „Die Befunde wollen umso sorgfältiger beachtet und weiter beobachtet werden, als die Bistumsblätter nach ihrem gegenwärtigen Selbstverständnis sowie nach der zur Zeit für sie geltenden pastoralen Zielsetzung nicht eigentlich Organe der Altenseelsorge sein wollen und sollen. Allerdings ist dieser Gedanke erwägenswert: Falls es sich in weiteren Untersuchungen herausstellen sollte, daß die Bistumsblätter zwar eine überalterte Leserschaft haben, daß diese Leserschaft aber (bei gleichbleibender Überalterungsstruktur) gleichmäßig nachwächst, wäre diese Erkenntnis sowohl für die Pastoral als auch für die Werbung (Abonnenten und Inserenten) ein wichtiger Gesichtspunkt.

Für den gegenwärtigen Zeitpunkt [1973] ergeben sich aus dieser Skizze als Folgerungen drei Vorschläge:

1. Herausgeber und Verleger der Bistumsblätter sollten darauf aufmerksam gemacht werden, daß die Beobachtung der Altersstruktur zur Zeit höchste Aufmerksamkeit verdient.

2. In die Feldbefragung [die damals schon vorbereitet wurde] sollten Fragen eingebaut werden, die die Rekonstruktion des AbonnementsEintrittsalters erlauben und damit eine mindestens partielle Rekonstruktion der Altersstruktur-Entwicklung.

3. Die beobachtete Parallelität von Auflagenschwund und Kirchenbesucherrückgang (Stellungnahme Nr. 7) läßt gemeinsame Ursachen vermuten: die ,ordentliche ${ }^{\prime}$ Kirche (im Unterschied zur außerordentlichen) ist im Begriff, eine Kirche der alten Menschen zu werden. Sofern die Kirchenleitung dies für ein drängendes Problem hält, sollten entsprechende pastoralsoziologische Untersuchungen, sofern sie eingeleitet werden, den kommunikationssoziologischen Aspekt, im besonderen den des Abonnentenverhaltens, mit einbeziehen. “

Die Stichworte Organe der Altenseelsorge und überalterte, aber bei gleichbleibender Überalterungsstruktur gleichmäßig nachwachsende Leserschaft haben damals wenig Beifall gefunden. Die mit ihnen angesprochene Eventualität ist übrigens nicht eingetreten. Denn heute, nach den Resultaten der Allensbach-Situationsanalyse, sieht die Grafik so aus: 


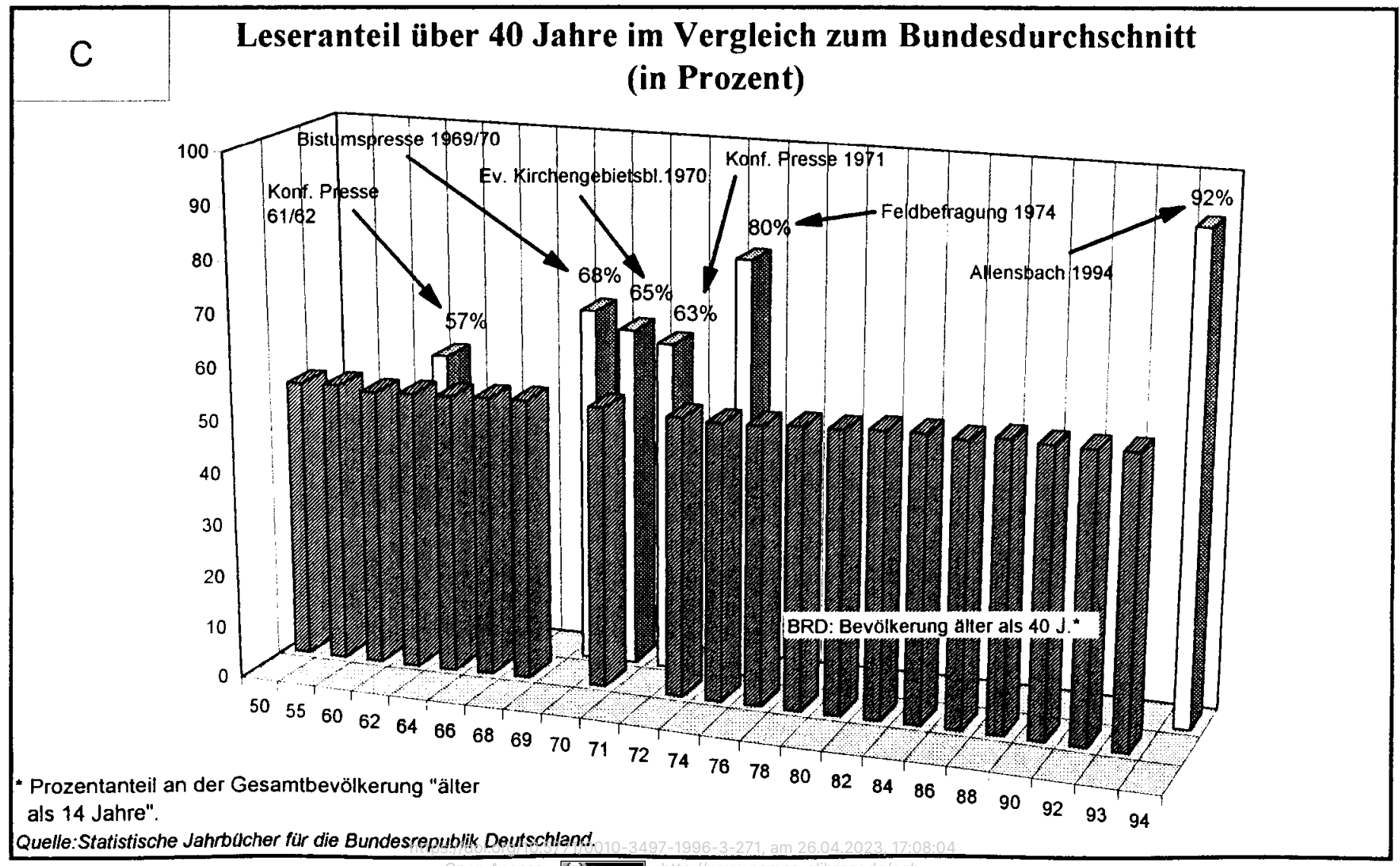

(co) EY 
Dieser Aspekt ist also, wie R. Schulz diplomatisch formuliert, „seither noch ungünstiger geworden“.12 1973 war es folgerichtig, wenn auch nicht gerade kreativ, vom Schreibtisch des Analytikers aus den Herausgebern und Verlegern der Bistumszeitungen die besonders aufmerksame Beobachtung der Altersstrukturentwicklung zu empfehlen.

Das Problem, das da hätte gelöst werden müssen, wurde erst in den Ergebnissen der "Feldbefragung“, also zwei Jahre später, so richtig deutlich: Allein mit Hilfe der Inhalte einer Kirchenzeitung ist die Schere zwischen den ermittelten Bedürfnissen der Alten und der Jungen nicht in den Griff zu bekommen. Um die Themenpräferenzen anschaulich darstellen zu können, habe ich in meiner Interpretation zur Feldbefragung ${ }^{13}$ die Gruppen "Jung" (unter 30 ) und "Alt" (50 und älter) gebildet. Dabei zeigte sich, daß bei den Jungen von den drei Spitzenthemen der Alten (Rang 1: Sichere Altersversorgung; 2: Versorgung im Krankheitsfall; 3: Probleme der alten Menschen) zwei (R1 und R3) überhaupt nicht unter die ersten zehn der Jungen gelangten; nur die Versorgung im Krankheitsfall schaffte es auf Rang 9 der Jungen, während umgekehrt Rang 1 der Jungen (Politische Entwicklung der BRD) immerhin Platz 7 der Alten belegte.

Thematische Polarisierungen dieses Typs waren damals reichlich aufzuweisen, und mit ihnen läßt sich bis heute nicht praktikabel umgehen: R. Schulz trifft in der Allensbacher Situationsanalyse die gleiche Problematik an und warnt ausdrücklich davor, "den "Altersspagat' zu überreizen". ${ }^{14}$

Spätestens an dieser Stelle ließe sich der Vorwurf erheben, es sei nicht fair, auf die Ergebnisse von damals mit dem Tenor zu verweisen, man habe alles schon vor 20 Jahren gewußt und obendrein auch noch Recht behalten. Der Vorwurf ist nicht ganz von der Hand zu weisen. Dennoch glaube ich, den Argumentationsgang so und nicht anders wählen zu müssen, denn es waren 1972/73 und spätestens 1975 einige Punkte erkannt und den Verantwortlichen auch mitgeteilt worden, die zu einer ernsteren Besinnung besonders in den Verlagen und Redaktionen hätten führen müssen, eben das Überalterungsphänomen und der "Altersspagat". Einer Institution, die sonst eher als konservativ gilt, kann man in diesem Punkt keinen Vorwurf machen: der Deutschen Bischofskonferenz. Sie hat mit der Gründung der Medien-Dienstleistung Gesellschaft eine Einrichtung geschaffen, die inzwischen sehr viel auch für die Bistumszeitungen getan hat und vielleicht noch mehr hätte tun können, wenn man in den Verlagen mit mehr Entschiedenheit neue Strategien entwickelt hätte. Stattdessen wurde mit immer neuen Jugendseiten, unrealistischen Beilagenprojekten und Geschenkabonne-

12 R. Schulz, Chancen (Anm. 7), 155.

13 Vgl. M. Schmolke, Kommentar (Anm. 4), $20 \mathrm{ff}$.

14 R. Schulz, Chancen (Anm. 7), 189. 
ments für junge Familien operiert. Aus der Allensbach-Studie geht inzwischen ziemlich deutlich hervor, was niemand auszusprechen gewagt hatte: Die Jugend will diesen Pressetyp nicht und braucht ihn nicht, - jedenfalls nicht so, daß sie dafür die feste Bindung eines Abonnements eingehen würde: Der Nutzen dieser wöchentlichen Lektüre erscheint $\mathbf{z u}$ klein, und die von ihr gebotene Gratifikation reißt - im Vergleich zum konkurrierenden Medienumfeld - niemanden vom Sessel.

Woran das unter anderem auch liegen könnte, erklären einige Ergebnisse der Inhaltsanalyse von H.M. Kepplinger und S.C. Ehmig. ${ }^{15}$ Im großen und ganzen aber scheint mir das Verhältnis zur Bistumszeitung sehr viel mit Bindungsbereitschaft und tatsächlicher Bindung zu tun zu haben.

\section{„Kirchlichkeit" und Bindung}

Die Bindung an ein kirchliches Wochenblatt ist im Kern nur dann sinnvoll, wenn es eine Bindung an die hinter dem Blatt stehende Institution, die Kirche, gibt.

Wie Hannes Burger einmal die Kündigung des Kirchenzeitungsabonnements als den "kleinen Kirchenaustritt" bezeichnet hat ${ }^{16}$, so kann heute umgekehrt die Aufrechterhaltung des Abonnements als das kleine In-der-Kirche-Bleiben interpretiert werden, als ein sekundärer Indikator für "Kirchlichkeit".

Mit diesem Begriff sind seit 1972 einige Anstrengungen verbunden, den Niedergang und die am Ende vielleicht doch mögliche Stabilisierung der Bistumszeitungen zu erklären.

Systematisch haben M.P. Becker und ich das in der oben ausgewerteten Stellungnahme Nr. 7 des Gutachtens Katholische Publizistik 72/73 getan, wobei wir, stark vereinfachend, den Besuch der Sonntagsmesse als einzigen Indikator heranzogen; damit befanden wir uns in guter Gesellschaft, denn einerseits sah Franz Groner "diese Meßzahl im Vergleich zu allen anderen Meßmöglichkeiten als besonders aufschlußreich für den jeweiligen Grad kirchlicher Bindung"17, und

15 H. M. Kepplinger/S. C. Ehmig, Form und Inhalt der Bistumspresse, in: CS 29 (1996) 206-233.

16 Der "kleine Kirchenaustritt", Titel einer Glosse von $H$. Burger, in: "Münchener katholischen Kirchenzeitung", 58 (1965) Nr. 25 vom 20.06.1965.

17 F. Groner, Integrationsschwund in der katholischen Kirche in Deutschland, in: Jahrbuch für christliche Sozialwissenschaften 12 (1971) 215-139, 215. 
andererseits operierte auch Gerhard Schmidtchen bei der Erstellung der großen Synoden-Umfrage damit. 18

Alle Interpretatoren und Kommentatoren von BistumspresseUntersuchungen vermieden es bislang jedoch, einen Kausalzusammenhang zwischen den drei sinkenden Kurven zu behaupten, also zwischen

- Rückgang der Kirchlichkeit, gemessen am sonntäglichen Gottesdienstbesuch,

- Sinken der Gesamtauflage der Bistumspresse und

- Sinken der Bezieherdichte.

Auch in der jüngsten Interpretation der Allensbach-Daten von 1994 warnt Renate Köcher ausdrücklich vor der Annahme, es bestehe ein monokausaler Zusammenhang:

„Angesichts des Gleichschritts der Entwicklungen der religiösen Kultur und der Resonanz kirchlicher Medien drängt sich die Frage auf, ob nicht die Kirchenzeitungen lediglich Opfer einer Abnahme kirchlicher Bindungen sind und unabhängig von der Qualität der redaktionellen Angebote zwangsläufig an Auflage verlieren müssen, wenn sich die Entkirchlichung der Gesellschaft weiter fortsetzt.

Die Ergebnisse der Situationsanalyse der Kirchenpresse stützen jedoch die These, daß sie nicht lediglich das Opfer externer Entwicklungen sind, sondern daß auch gravierende Defizite vorliegen, die behoben werden können.

Die Probleme der Bistumspresse gehen keineswegs ausschließlich auf die Schwächung religiöser und kirchlicher Bindung zurück."19 Es gebe vielmehr weitere Ursachen, nämlich die generell nicht mehr ausreichende Tragfähigkeit des Lesemotivs Weltanschauung bei der Weltanschauungspresse schlechthin (Ende der Parteizeitungen), die Bezugsebene (nur Gemeinde und Weltkirche sind interessant), die Knappheit der Zeit und der Aufmerksamkeit unter dem Druck der Medienkonsumkonkurrenz, die Entwicklung zum Zielgruppen-Marketing und schließlich das Nicht-Mithalten-Können im Styling der Produkte.

Andererseits verblüfft die Parallelität der Niedergangskurven. In der Graphik, die ich meiner 1993 publizierten Anfrage zum "Anfang der Talsohle ${ }^{\prime 20}$ beigegeben hatte, wollten es die eher zufällig gewählten Maßstäbe für Gesamtauflage und Gottesdienstbesucher-Anteil, daß diese beiden Kurven ab 1989 absolut parallel (auf einer Spur!) verliefen;

18 G. Schmidtchen, Zwischen Kirche und Gesellschaft, Freiburg/Br. 1972, 94ff.

19 R. Köcher, Religiöse Kultur und Kommunikation, in: CS 29 (1996) 145-152, 149.

20 Vgl. M. Schmolke, Anfang der Talsohle (Anm. 10). 
eine Fortschreibung würde diese Eigenart bestätigen: Der Gottesdienstbesucher-Anteil war bis 1993 nach amtlicher kirchlicher Statistik ${ }^{21}$ auf 19,3 \% (1990: $22 \%$ ) gesunken, die Auflage auf 1,39 Mio. (1990: 1,46 Mio.) und die Bezieherdichte auf 4,96 (1990: 5,3). Es ist also schwer, die Satire (Gemeinsam bergab!) nicht zu schreiben, obwohl die Parallelität als solche in der Tat kein Náchweis für kausale Zusammenhänge ist.

Für die Annahme, daß es diese dennoch gibt, spricht z.B. folgender Befund von R. Schulz: „Die Situationsanalyse von 1994 hat auch Schmolkes Aussage bestätigt, daß ,Kirchenpresse, speziell Bistumspresse vorwiegend kircheninterne Kommunikation ist. Sie erreicht hauptsächlich Kirchgänger'. Wie schon 1975 werden auch heute mit der Bistumszeitung ganz überwiegend regelmäßige Gottesdienstbesucher erreicht (1975: $83 \%, 1994: 79 \%) .{ }^{222}$

Gegen die Annahme eines kausalen Zusammenhanges sprechen vor allem die differenzierenden Argumente von Renate Köcher in Heft 2/1996 dieser Zeitschrift. Im Unterschied zu nicht wenigen anderen Autoren, die unseren Zeitgenossen ein erhebliches Quantum an (diffusen) religiösen Interessen und sogar Bedürfnissen zu bestätigen nicht müde werden (Esoterik-Boom, Sekten), stellt Köcher nüchtern, aber offensichtlich gestützt auf Allensbach-Ergebnisse, fest: „Die häufig kolportierte These, die Erosionserscheinungen beträfen ausschließlich die Institution Kirche, nicht die Religiosität der Bevölkerung, hält der empirischen Überprüfung nicht stand. Die Zusammenhänge zwischen kirchlichen Bindungen und individueller Glaubenskraft sind unverändert denkbar eng. In den Bevölkerungskreisen mit engen kirchlichen Bindungen definieren sich $96 \%$ als religiös, von den kirchlich Distanzierten ganze $16 \%$. In der verharmlosenden Diagnose einer isolierten Kirchenkrise spiegelt sich die allgemeine Unterschätzung der Bedeutung von Institutionen." 23

Gegen die Annahme eines einfachen kausalen Zusammenhanges spricht aber auch die Evidenz sehr aktiven Gemeindelebens bei gleichzeitiger Distanz zu den offiziellen Einrichtungen der Diözese. Offensichtlich sind nicht wenige Pfarrgemeinden „näher beim Zweiten Vaticanum“ zu bleiben bemüht, während es in den Großstrukturen

21 Also nicht nach den Erhebungsdaten, wie wir sie in "Chancen für die Bistumszeitungen 1994" (vgl. Anm. 5), z.B. in Tabelle A1 finden, sondern nach den vom Referat Statistik des Sekretariats der Deutschen Bischofskonferenz für 1993 publizierten Daten.

22 R. Schulz, Chancen (Anm. 7), 155. - Der Rückgang des Anteils der Bistumsblatt-Bezieher unter den Kirchgängern beträgt von 1975 bis 1994 nur vier Prozentpunkte und täuscht damit Stabilität vor; die absoluten Zahlen sind da deutlicher: 1975 gab es noch gut 9 Mio. Sonntagsmeß-Besucher (nur West), 1993 nur mehr 5,4 Mio. gesamtdeutsch. 
jedenfalls einiger deutschsprachiger Diözesen eine Art Roll-back gegeben hat, verursacht u.a. durch die Personalpolitik des Vatikans.

In der Erzdiözese Salzburg hat sich 1994/95, ausgelöst durch Differenzen zwischen dem Erzbischof einerseits und dem Chefrełakteur sowie dem geistlichen Beirat der diözesanen Kirchenzeitung andererseits, die schließlich in ein Machtwort des Erzbischofs (Entfernung der beiden publizistisch Verantwortlichen) mündeten, eine ernste Krise des "Rupertusblatts" entwickelt: Es verlor innerhalb eines Jahres 8.000 (von vorher 28.000) Abonnenten, also $28 \%$ seiner zahlenden Klientel. Die Beendigung des Abonnements ist hier jedoch nicht der kleine Kirchenaustritt, sondern die in gewissen Stilmerkmalen "protestartische" Demonstration besonderer Kirchennähe bei gleichzeitiger Hirtenferne.

Die Tatsache, daß es in wahrscheinlich nicht wenigen Gemeinden ein quantitativ 'nennenswertes Potential kirchenverbundeneri, aber protestierenden Lebens gibt (Kirchenvolksbegehren in Österreich und Deutschland!), läßt mindestens die Hypothese denkbar erscheinen, daß zwar "Kirchlichkeit" im konventionellen Sinne und BistumsblattAbonnement zusammengehen (und auch parallel zu Tal fahren), daß es aber außerhalb dieser Zone ein sich selbst ernst und wichtig nehmendes Gemeindeleben gibt, das des ebenfalls konventionellen Bistumsblatts besonders dann nicht bedarf, wenn es wieder in den ïberholt geglaubten Typ „Sprachrohr des Bischofs“ zurückfällt, - eine Auffassung, die von der Allensbacher Situationsanalyse einmal mehr kestätigt wird. ${ }^{24}$

\section{Bistumsblatt-Leser in Distanz zum Bistum}

Der Eindruck der Entfernung (Entfremdung?) mancher Gemeinden von der diözesanen Zentrale korrespondiert mit den relativ schwachen Interessen-Rängen, die der Themenkomplex Diözese bei Abonnenten/ Lesern wie Fernerstehenden sowohl in der "Feldbefragung" als auch in der Allensbacher Situationsanalyse erzielt hat.

Zum Thema „Veranstaltungen im Bistum“ sagten 1974 (nur) $24 \%$ der Bezieher und $21 \%$ der Katholiken allgemein, es sei "besonders interessant", das Thema "Veranstaltungen in meiner Pfarrgemeinde" hielten dagegen $40 \%$ der Bezieher und $43 \%$ der Katholiken allgemein für "besonders interessant". Dazu kommt, daß nach Meinung von $92 \%$ der Bezieher über Veranstaltungen im Bistum ausreichend berichtet wurde, während nur $68 \%$ die Pfarrgemeinde ausreichend abgedeckt sahen. ${ }^{25}$

24 Bd I, 50 und 54.

25 Feldbefragung (vgl. Anm. 4), Teil A, Bd. I, 71f. 
Die Unterschiede sind signifikant, wenn auch wohl nicht entscheidend für die Lebenslinie der Bistumsblätter. Aber es gibt doch zu denken, daß die Auswerter der Situationsanalyse 1994/95, nachdem inzwischen eine Flut von Pfarrbriefen, Gemeindeblättern etc. entstanden war, auf den gleichen Befund wie 1974 stoßen; R. Schulz spricht sogar von einer "überraschend geringen Bedeutung des Bistums als Identifikations- und Interessenebene für viele Katholiken"2s und sagt an anderer Stelle:

„Für die meisten Katholiken ist die eigene Kirchengemeinde die eigentliche Identifikations- und Interessenebene. Die Bindung an das Bistum ist deutlich geringer, was allerdings beachtliches Interesse schon der ab 40jährigen - an Stellungnahmen und Aussagen des Bischofs nicht ausschließt." 27 Oder anders pointiert: „Ausgesprochen gering ist dagegen das Interesse der Katholiken an Beiträgen ,Aus dem Leben des Bistums' $(14 \%)^{\prime \prime} .28$

Hier wird erkennbar, daß die Diözese für viele Katholiken keine geeignete Identifikations- bzw. Interessenebene darstellt, so daß die Beziehung bzw. das Selbstverständnis als Zeitung des Bistums bzw. des Bischofs die Beachtungs- und Verbreitungschancen in Teilen des Leserpotentials hemmen kann. Die Leser der Kirchenzeitungen wie auch die Katholiken insgesamt interessieren sich in erster Linie für die ,Kirche', für Stellungnahmen zu Glaubensfragen und wichtigen gesellschaftlichen Themen sowie für die eigene Kirchengemeinde, im Nahbereich, dagegen nur wenig für die Bistumsebene. ${ }^{29}$

Die Bistumszeitungen sehen sich hier einem für die regionale und lokale Presse allgemein geltenden Phänomen gegenüber ${ }^{30}$, und die Verantwortlichen der Diözesen werden über diesen für sie schmerzli-

26 R. Schulz, Chancen (Anm. 7), 200.

27 Ebd., 177.

28 Ebd., 169.

29 Ebd., 169.

30 Vgl. W. J. Schütz: Lokalkommunikation - Zustand und Zukunft, in: Presse-, Rundfunk- und Filmarchive - Mediendokumentation 2, München 1980, 165-171, hier 170: "... der Raum örtlichen Erlebens ist für die Leser relativ gering, und wir dürfen uns das Interesse des Lesers, des Hörers graphisch so vorstellen, daß das Lokale an der Basis eines Kegels angesiedelt ist und sich das Interesse zur Spitze hin - also mit zunehmender Entfernung vom Ort, seiner Umwelt - rasch verringert. Stellen wir uns einen weiteren Kegel vor, der umgekehrt auf der Spitze des ersten Kegels steht, kann die nationale und internationale Politik vom Leser- und Hörerinteresse her wieder ganz oben, also am breiten Kegelfuß angesetzt werden. Vom Rezipienten her bleibt somit für das Regionale - um kommunikativ wirksam zu sein - wenig oder nichts ..." 
chen Befund nachdenken müssen; dabei ist vor übereilten Beschlüssen zu warnen. ${ }^{31}$

In jedem Falle ist die Einbeziehung der Bischöfe in die Datenerhebung der Allensbacher Analyse und die Zusammenarbeit mit ihnen bei der Umsetzung der Ergebnisse $^{32}$ ein Faktum, das auf konstruktive Entwicklungen hoffen läßt.

Die Auswertung der Position der Bischöfe ist wie so manches andere Teil-Resultat eine im Rahmen dieser Sekundär-Kommentierung noch zu leistende Aufgabe, und für den Autor stellt es ganz allgemein eine Versuchung dar, jetzt noch in die Diskussion einzelner wesentlicher Punkte der von R. Schulz vorgelegten Interpretation einzutreten, dies umso mehr, als der von Schulz im vorigen Heft von Communicatio Socialis publizierte Text bereits Kondensat und Akzentuierung zugleich ist. Angesichts dieser Gegebenheit hieße es, bei jedem Ansatz einer Sekundär-Interpretation zur Quelle selbst (vgl. Anm. 5) zurückzukehren. Das würde zwangsläufig auch dann in eine zweite Expertise 33 münden, wenn meine Interpretationsergebnisse in den meisten Fällen mit jenen von Schulz - und womöglich überwiegend auch mit den „Empfehlungen“ des Bandes I der Analyse - übereinstimmen würden.

Womit ich mich ebenso wie mit der oben angesprochenen Position der Bischöfe erst nach weiterer gründlicher Überlegung auseinandersetzen möchte, ist die von Kepplinger und Ehmig erstellte und kommentierte Inhaltsanalyse. ${ }^{34}$ Sie liest sich, wie bittere Medizin schmeckt. $\mathrm{Zu}$ schlucken hatten aber wohl nicht nur die Redaktionen, sondern auch die Verantwortlichen der von Funktionsverlust bedrohten Full-Service-Agentur Kirche.

31 In der Erzdiözese Wien scheint die Verwirklichung eines solchen Beschlusses bevorzustehen: die Gründung einer "Mitgliederzeitung", die künftig elfmal jährlich an alle katholischen Haushalte kostenlos verschickt werden soll. Die Folgen für die "Wiener Kirchenzeitung", die noch mit 35.000 Exemplaren verkauft wird, kann man sich ausmalen. Vgl. „APA-Journal Medien” vom 03.07.1996, 4, sowie „AKP Mitteilungen” 2/1996, 18: „Der Leiter des [Wiener] Erzbischöflichen Amts für Öffentlichkeitsarbeit und Kommunikation, Wolfgang Bergmann, erklärte, die Kirche müsse von sich aus an die Menschen herantreten. Sie solle nicht von den Katholiken verlangen, eine Zeitung zu kaufen, um etwas aus ihrer Kirche zu erfahren. Bergmann ließ zunächst offen, ob das neue Magazin das Ende der ,Wiener Kirchenzeitung' bedeute. Es solle keine ,Werbepostille, sondern eine ,Dienstleistung' sein." Vgl. H. Klinge, Hilfen zur Umsetzung (Anm. 3).

Eine solche bedürfte einer besonderen Beauftragung.

Sie beruht auf dem Band III (Form und Inhalt der Bistumszeitungen) der Gesamtuntersuchung "Chancen für die Bistumszeitungen 1994". 


\section{SUMMARY}

In 1994 the situation of the Catholic weeklies edited by the dioceses of Germany ("Bistumszeitungen") has been analysed by the renowned "Institut für Demoskopie Allensbach" (see "Communicatio Socialis", no. 2:1996). The reason for starting another analysis was the continuing decline of the circulation. The weekly circulation of the 25 papers decreased from 2.45 million copies in 1963 to 1.29 million copies in 1995. The recent Allensbach-Analysis (commissioned by the „Medien Dienstleistung [Media Service] Company MDG) completes a series of about five comparable studies since 1970. The results can be compared under different main critical aspects: 1 . The contents offered by the bishops' iveeklies do no longer meet the needs and expectations of the audiences. 2. Concerning the strata of age groups the audiences are not comparable with the Catholic section of the German population. The readers have grown too old. 3. The graph of decline of circulation parallels the graph of the shrinking number of people attending the sunday services. Inspite of the fact that most of the defects and dysfunctions have been discovered 20 years ago there are no indicators of new strategies developed to finish the never ending crisis. The weeklies are going to loose the remaining audience (1.29 million subscribers, i.e. 3 million readers) unless the publishers and editorial staffs follow the strategies of reform presented by the MDG's head Heiko Klinge in "Communicatio Socialis" no. 2:1996.

\section{RÉSUMÉ}

La situation des hebdomadaires des diocèses allemands („journaux de diocèses") a été analysée en 1994 par l'institut de science de l'opinion Allensbach (voir "Communicatio Socialis" Nr. 2/1996). La raison pour laquelle l'analyse a été effectuée est la baisse constante du tirage de ses hebdonıadaires: Le tirage de l'année 1995 ne représentait que la moitié de celui de 1963. (Baisse de 2,45 millions à 1,29 millions d'exemplaires par semaine). La récente analyse de la situation fait partie de tout un ensemble d'analyses comparables depuis 1970. Les résultats ont toujours été les mêmes: 1) L'offre de lecture des journaux ne correspond plus de façon suffisante aux attentes des abonnés. 2) La structure d'âge des lecteurs n'est pas favorable (trop âgés) et ceci s'accentue d'analyse en analyse. 3) Si l'on fait uns courbe de la diminution du nombre des abonnés et des lecteurs, on s'aperçoit qu'elle est parallèle à la courbe des liens décroissants envers l'eglise (baisse du nombre des pratiquants). Une amélioration n'est pas perceptible bien que certains défauts aient été perçus depuis déjà une vingtaine d'années. La récente analyse montre clairement certains défauts et fait des propositions pour surmonter la crise. Il faut espérer que l'on suivra ces propositions afin que l'on ne perde pas plus de lecteurs.

\section{RESUMEN}

La situación de los semanarios de las diócesis alemanas ("periódicos diocesanos") fue analizada en 1994 por el Instituto de Demoscopía de Allensbach (ver $\mathrm{N}^{\circ}$ 2/1996 de "Communicatio Socilias"). La razón de este análisis fue continua disminución de las tiradas desde 1964. La totalidad de la edición en 1995 alcanzó a la mitad de la de 1963 (disminución de 2,45 millones a 1,29 millones de ejemplares por semana). Este nuevo análisis se sitúa junto a una serie de investigaciones comparables desde 1970. Los resultados a lcs que se ha llegado son muy parecidos: $1^{\circ}$ la oferta de lertura que se ofrece no corresponde suficientemente a las espectativas de los suscritos. $2^{\circ}$ la estructura de edad de los lectores no es favorable (demasiada edad) y de investigación a investigación se hace aún más desfavorable. $3^{\circ}$ La disminución de los 
suscriptores y de los lectores se puede representar en una curva que transcurre paralela a la curva de la disminución de la ligazón a la iglesia (dísminución de las visitas a los servicios religiosos). No se vislumbra una mejoría a pesar de que algunas de las carencias son conocidas ya desde hace 20 años. El último análísis muestra de forma clara las deficiencias y hace proposiciones para lograr una superación de la crisis. Es de esperar que estas proposiciones sean seguidas, para que no se pierda la aún gran cantidad de lectores. 